\title{
Introduction: 3rd Pediatric Body MRI Course supplement
}

\author{
Govind B. Chavhan ${ }^{1,2}$ (D) Jonathan R. Dillman ${ }^{3}$
}

Received: 22 June 2021 / Revised: 22 June 2021 / Accepted: 12 July 2021 / Published online: 30 July 2021

(C) The Author(s), under exclusive licence to Springer-Verlag GmbH Germany, part of Springer Nature 2021

It is said that necessity is the mother of invention. Well, we did not invent anything by conducting the Society for Pediatric Radiology's 3rd Pediatric Body MRI Course virtually, but we did face the formidable challenge of running a virtual, entirely live 3-day meeting for the first time and during a ravaging pandemic. Our anticipated live in-person meeting was converted into a virtual meeting with only $\sim 2.5$ months to prepare. We took calculated risks based on guidance and leadership provided by Society for Pediatric Radiology (SPR) Board Chair Dr. Chris Cassady, who not only pushed us but inspired us to take on this challenge. Angela Davis and Jennifer Raju from the SPR left no stone unturned, demonstrating relentless hard work, dedication and passion. We worked out the intricacies of a virtual meeting, including identifying what could possibly go wrong and how to prevent it. The end result was a well-received educational course attended by 434 keenly interested delegates from 42 countries. In the end, we were true to Mark Twain's version of the mantra: necessity is the mother of taking chances!

Key to the success of this course was the wealth of expertise, experience and hard work of all of the speakers. This virtual course, conducted Sept. 25-27, 2020, featured 45 faculty presenting 48 lectures, including case-based reviews and a session of lectures done TED-Talk style. The course also included a session on advanced MRI techniques in collaboration with the International Society for Magnetic Resonance in Medicine (ISMRM). Participating faculty included a pediatric hepatologist, a gastroenterologist with expertise in pediatric pancreatic diseases, a pediatric gynecologist, an oncologist

Govind B. Chavhan

drgovindchavhan@yahoo.com

1 Department of Diagnostic Imaging, The Hospital for Sick Children, 555 University Ave., Toronto, ON M5G 1X8, Canada

2 Medical Imaging, University of Toronto, Toronto, ON, Canada

3 Department of Radiology, Cincinnati Children's Hospital Medical Center, University of Cincinnati College of Medicine,

Cincinnati, OH, USA and an interventional radiologist treating vascular anomalies, all of whom provided clinical perspectives before each session.

The program included basic, advanced and quantitative MRI techniques applied to body imaging, with in-depth coverage of MR imaging related to various pediatric body systems including hepatobiliary, genitourinary, bowel and chest, along with vascular anomalies and oncological imaging. The course also covered topics requiring discussion and debate in the TED-Talk-like sessions. The conference included $18.5 \mathrm{~h}$ of American Medical Association Physician's Recognition Award (AMA PRA) Category 1 Credits for physicians and $20.5 \mathrm{~h}$ of American Registry of Radiologic Technologists (ARRT) Category A credits for technologists. The course also offered five self-assessment modules (SAM), for 5.5 SAM credits.

This special supplement of Pediatric Radiology includes review articles based on the talks presented at the course to further spread the benefits of the course to the SPR membership and broader radiology community. We cannot thank enough to the speakers who worked very hard to produce high-impact presentations and high-quality review papers for this special issue. We would like to thank Dr. Peter Strouse, co-editor of Pediatric Radiology, for his guidance for this supplement and Ms. Stephanie Custer for keeping us coordinated. It is our hope that this special issue will serve as an excellent reference source for radiologists involved in the diagnosis and management of children.

Publisher's note Springer Nature remains neutral with regard to jurisdictional claims in published maps and institutional affiliations. 